\title{
Large-scale lipidomic profiling identifies novel potential biomarkers for prion diseases and highlights lipid raft-related pathways
}

Yong-Chan Kim ${ }^{1,2}$, Junbeom Lee ${ }^{3}$, Dae-Weon Lee ${ }^{3,4^{*}}$ and Byung-Hoon Jeong ${ }^{1,2^{*}}$ (D)

\begin{abstract}
Prion diseases are transmissible spongiform encephalopathies induced by the abnormally-folded prion protein ( $\mathrm{PrPS}^{\mathrm{Sc}}$ ), which is derived from the normal prion protein $\left(\operatorname{Pr} \mathrm{PC}^{\mathrm{C}}\right.$ ). Previous studies have reported that lipid rafts play a pivotal role in the conversion of $\mathrm{PrP}^{\mathrm{C}}$ into $\mathrm{PrP}^{\mathrm{SC}}$, and several therapeutic strategies targeting lipids have led to prolonged survival times in prion diseases. In addition, phosphatidylethanolamine, a glycerophospholipid member, accelerated prion disease progression. Although several studies have shown that prion diseases are significantly associated with lipids, lipidomic analyses of prion diseases have not been reported thus far. We intraperitoneally injected phosphatebuffered saline (PBS) or ME7 mouse prions into mice and sacrificed them at different time points (3 and 7 months) post-injection. To detect PrPSc in the mouse brain, we carried out western blotting analysis of the left hemisphere of the brain. To identify potential novel lipid biomarkers, we performed lipid extraction on the right hemisphere of the brain and liquid chromatography mass spectrometry (LC/MS) to analyze the lipidomic profiling between non-infected mice and prion-infected mice. Finally, we analyzed the altered lipid-related pathways by a lipid pathway enrichment analysis (LIPEA). We identified a total of 43 and 75 novel potential biomarkers at 3 and 7 months in prion-infected mice compared to non-infected mice, respectively. Among these novel potential biomarkers, approximately $75 \%$ of total lipids are glycerophospholipids. In addition, altered lipids between the non-infected and prion-infected mice were related to sphingolipid, glycerophospholipid and glycosylphosphatidylinositol (GPI)-anchor-related pathways. In the present study, we found novel potential biomarkers and therapeutic targets of prion disease. To the best of our knowledge, this study reports the first large-scale lipidomic profiling in prion diseases.
\end{abstract}

Keywords: Prion, Lipid, Lipidomics, Biomarker, Pathway, Lipid raft, GPI, LIPEA

\section{Introduction}

Prion diseases are fatal and irreversible neurodegenerative disorders caused by the abnormally folded prion protein $\left(\mathrm{PrP}^{\mathrm{Sc}}\right)$, which originates from the normal prion protein $\left(\operatorname{PrP}^{C}\right)$ [1-6]. Under normal physiological conditions, $\operatorname{PrP}^{\mathrm{C}}$ is located in lipid rafts via

\footnotetext{
*Correspondence: daeweonlee@ks.ac.kr; bhjeong@jbnu.ac.kr

1 Korea Zoonosis Research Institute, Jeonbuk National University, Iksan, Jeonbuk 54531, Republic of Korea

${ }^{3}$ Metabolomics Research Center for Functional Materials, Kyungsung University, Busan 48434, Republic of Korea
}

Full list of author information is available at the end of the article glycosylphosphatidylinositol (GPI)-anchoring and plays essential roles in signal transduction, neuroprotection and metal ion homeostasis [7-9]. However, under pathological conditions, $\operatorname{PrP}^{C}$ is converted into $\operatorname{PrP}^{\mathrm{Sc}}$. $\operatorname{PrP}^{\mathrm{C}}$ conversion may induce a loss of innate physiological functions but also confers deleterious effects in the host CNS and induces spongiform vacuolation and astrocytosis $[10-12]$.

Previous studies have reported that the lipid raft, which consists of glycosphingolipids, cholesterol and protein receptors organized in lipid microdomains, is critically associated with the conformational conversion process of original author(s) and the source, provide a link to the Creative Commons licence, and indicate if changes were made. The images or other third party material in this article are included in the article's Creative Commons licence, unless indicated otherwise in a credit line to the material. If material is not included in the article's Creative Commons licence and your intended use is not permitted by statutory regulation or exceeds the permitted use, you will need to obtain permission directly from the copyright holder. To view a copy of this licence, visit http://creativecommons.org/licenses/by/4.0/. The Creative Commons Public Domain Dedication waiver (http://creativeco mmons.org/publicdomain/zero/1.0/) applies to the data made available in this article, unless otherwise stated in a credit line to the data. 
$\operatorname{PrP}^{C}$ into $\operatorname{PrP}^{\mathrm{Sc}}[13,14]$. An in vitro prion disease model showed impaired cholesterol metabolism, and the reduction in cellular cholesterol levels by lovastatin, filipin and squalestatin contributed to diminishing $\operatorname{PrP}^{\mathrm{Sc}}$ formation [15-18]. In addition, mislocalization of $\operatorname{PrP}^{\mathrm{C}}$ by replacement or removal of the GPI anchor inhibited the conversion of $\operatorname{PrP}^{C}$ into $\operatorname{PrP}^{S c}[19,20]$. Strikingly, a recent study reported that phosphatidylethanolamine acts as an independent cofactor for in vitro $\operatorname{PrP}^{\mathrm{Sc}}$ formation in the absence of genetic materials [21]. Although several studies have suggested that lipids are significantly related to the conversion process of $\operatorname{Pr} \mathrm{P}^{\mathrm{Sc}}$, lipidomic profiling has not been reported in prion diseases thus far.

In the present study, we performed a lipidomic analysis in a mouse model of prion disease and identified novel lipid biomarkers of prion disease and altered lipidrelated pathways. For this, wild-type mice were injected intraperitoneally with phosphate-buffered saline (PBS) and mouse-adapted ME7 scrapie prions and sacrificed at different time points (3 and 7 months) post-injection. In addition, we carried out western blotting analysis to detect $\mathrm{PrP}^{\mathrm{Sc}}$ in prion-infected mice. Furthermore, we performed liquid chromatography mass spectrometry (LC/MS) and analyzed the lipidomic profiling between non-infected mice and prion-infected mice to identify novel lipid biomarkers for prion disease. Finally, we analyzed the altered lipid-related pathways by a lipid pathway enrichment analysis (LIPEA).

\section{Materials and methods}

\section{Ethical statements}

The mouse ME7 scrapie strain was obtained from The Roslin Institute, The University of Edinburgh. C57BL/6J mice were purchased from Nara Biotech (Pyeongtaek, Korea). All efforts were made to minimize the number of animals used and their suffering. All experimental procedures were accredited by the Institute of Animal Care and Use Committee of Jeonbuk National University (JBNU 2020-080).

\section{Inoculation of mice to investigate lipidomic profile}

Six C57BL/6 mice (6-week old) were inoculated by intraperitoneal injection with $100 \mu \mathrm{L}$ of $1 \%(\mathrm{w} / \mathrm{v})$ brain homogenate prepared from terminally ill ME7-infected mice. Post-inoculation, the mice were sacrificed at 3 $(n=3)$ and 7 months $(n=3)$. As control, six C57BL/6 mice (6-week old) were inoculated via the intraperitoneal route with $100 \mu \mathrm{L}$ of PBS. Post-inoculation, the mice were sacrificed at $3(n=3)$ and 7 months $(n=3)$. Prion disease symptoms including ataxia, tail rigidity and kyphosis were observed in prion-infected mice at 7 months postinjection. The incubation period of ME7-infected mice was $227 \pm 1$ days $(n=3)$.

\section{Western blotting analysis to detect $\operatorname{PrP}^{\mathrm{Sc}}$}

The brains were extracted and homogenized with $10 \%$ volumes of RIPA lysis buffer (Thermo Fisher Scientific, USA) containing a cocktail of protease inhibitors (Roche, Germany). To detect specifically $\mathrm{PrP}^{\mathrm{Sc}}$ in the mouse brain, $40 \mu \mathrm{g} / \mathrm{mL}$ proteinase $\mathrm{K}$ was added for $1 \mathrm{~h}$ at $37^{\circ} \mathrm{C}$. The samples were heated to $95^{\circ} \mathrm{C}$ for $10 \mathrm{~min}$ before being loaded into each lane in a $12 \%$ sodium dodecyl sulfate (SDS)-polyacrylamide gel with 5X sample buffer (Thermo Fisher Scientific, USA). The loaded proteins were transferred to a nitrocellulose membrane (Amersham, USA) using an electrophoretic transfer system (BioRad, USA) at $100 \mathrm{~V}$ for $1.5 \mathrm{~h}$. The membranes were washed with a Tris-buffered saline solution ( $\mathrm{pH}$ 7.6) containing $0.05 \%$ Tween 20 (TBST) and then blocked in TBST containing 5\% skim milk (Santa Cruz Biotechnology, USA) for $1.5 \mathrm{~h}$ at room temperature. The membranes were then incubated at $4{ }^{\circ} \mathrm{C}$ overnight with mouse monoclonal antiPrP antibody SAF84 (1:200; Cayman, USA) [22]. After washing in TBST, the membranes were incubated with horseradish peroxidase-conjugated secondary antibodies (Sigma-Aldrich, USA) for $1 \mathrm{~h}$ and washed in TBST again. The target protein bands were visualized by autoradiography using a Pierce ECL kit (Thermo Fisher Scientific, USA).

\section{Lipid extraction}

Lipid extracts were prepared by the modified Bligh and Dyer method as described in a previous study [23]. In brief, each sample was treated with $3 \mathrm{~mL}$ of solution (2:1, $\mathrm{MeOH}$ : chloroform, $\mathrm{v} / \mathrm{v}$ ) and then shaken and incubated for $20 \mathrm{~min}$. One milliliter of chloroform and $1.8 \mathrm{~mL}$ of water were added to the sample and vortexed for $30 \mathrm{~s}$. The lower layer was obtained by centrifugation $(1750 \mathrm{~g})$ for $10 \mathrm{~min}$ at $10{ }^{\circ} \mathrm{C}$, transferred to a new tube and dried under $\mathrm{N}_{2}$. Finally, all dried samples were reconstituted with $\mathrm{MeOH} /$ chloroform $(200 \mu \mathrm{L}, 1: 1)$ and treated by ultrasound for $5 \mathrm{~min}$. Then, the clear solution was used for LC/MS analysis after centrifugation $(1750 \mathrm{~g})$ for $10 \mathrm{~min}$ at room temperature. The recovery rates of lipid standards (SPLASH ${ }^{\circledR}$ LIPIDOMIX ${ }^{\circledR}$ Mass Spec Standard, Avanti polar lipids, UK) were checked to confirm the efficiency of lipid extraction for later unbiased lipid analysis, and the results showed more than $50 \%$ recovery rates.

\section{Lipid analysis using LC/MS}

A liquid chromatograph triple-quadrupole mass spectrometer (Agilent Technologies, USA; Metabolomics Research Center for Functional Materials, Kyungsung University) with an electrospray ion source (ESI) was utilized in the present study. Chromatic separation was achieved using an XSelect CSH C18 Column $(3.5 \mu \mathrm{m}$, 
$4.6 \mathrm{~mm}$ X $100 \mathrm{~mm}$, Waters, USA). The column temperature was set at $55{ }^{\circ} \mathrm{C}$. A binary mobile phase system was described as follows: mobile phase A: acetonitrile/water (60:40) with $10 \mathrm{mM}$ ammonium formate and $0.1 \%$ formic acid; and mobile phase B: Isopropanol/acetonitrile (90:10) with $10 \mathrm{mM}$ ammonium formate and $0.1 \%$ formic acid. The mobile phase constitution was changed as follows: initiation at $40 \% \mathrm{~B}$ followed by a linear gradient to $43 \%$ B over $2 \mathrm{~min}, 50 \% \mathrm{~B}$ at $2.1 \mathrm{~min}, 54 \% \mathrm{~B}$ at $12 \mathrm{~min}, 70 \%$ $\mathrm{B}$ at $12.1 \mathrm{~min}, 99 \% \mathrm{~B}$ at $18 \mathrm{~min}$, and $40 \% \mathrm{~B}$ at $18.1 \mathrm{~min}$ for $1.9 \mathrm{~min}$. The flow rate was set at $400 \mu \mathrm{L} / \mathrm{min}$. The injection volume was $20 \mu \mathrm{L}$. The mass spectrometry analysis was performed using ESI in negative and positive modes. The capillary voltages were set under the negative and positive modes as follows: $2.0 \mathrm{kV}$ (for positive) and $1.0 \mathrm{kV}$ (for negative).

\section{Data processing}

Mass Profiler Professional software (Agilent Technologies, USA) was employed to visualize, process and interpret multidimensional LC/MS data. Data were further normalized using total ion intensity. Analyses were performed based on multivariate statistical methods, including principal component analysis. The peak height intensity of the differential lipid metabolites was compared by a two-way analysis of variance (ANOVA) using statistical software to confirm the biomarker alterations between non-infected mice and prion-infected mice. Differentially up- or downregulated lipids between these two groups were defined as changes in lipids with values of $\left|\log _{2} \mathrm{FC}\right|>1$ and $P<0.05$.

\section{Pathway analysis}

To carry out the pathway analysis, a LIPEA [24] based on a database source including Kyoto Encyclopedia of Genes and Genomes (KEGG) was used to visualize relevant pathways of potential lipid biomarkers.

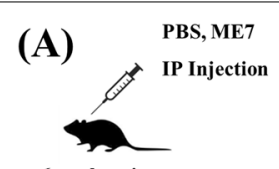

6 weeks mic

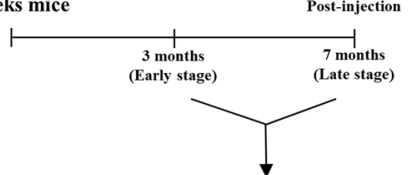

Sacrifice

Brain extraction

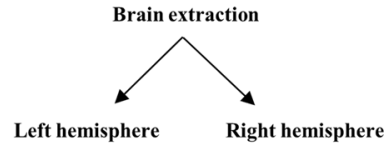

$\begin{array}{cc}\text { Left hemisphere } & \text { Right hemisphere } \\ \text { (Protein extraction) } & \text { (Lipid extraction) }\end{array}$

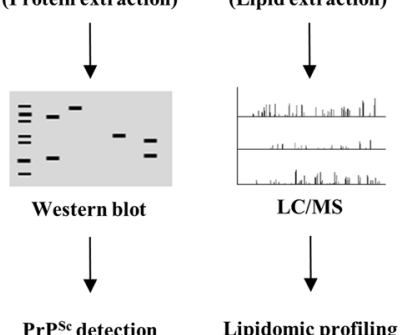

(B)

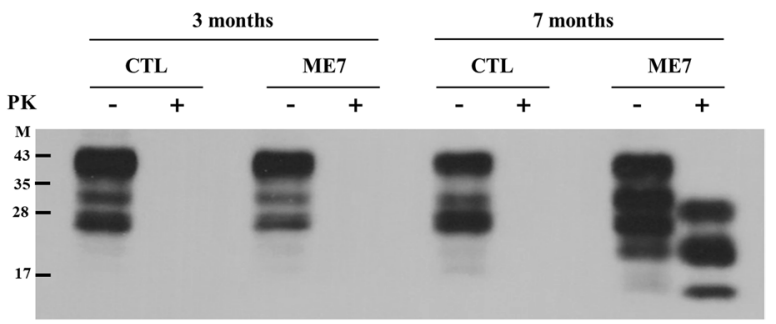

Figure 1 Lipidomic profiling from prion-infected mice. A Overall workflow for $\mathrm{Pr}^{\mathrm{SC}}$ detection and lipidomic profiling from prion-infected mice. IP: intraperitoneal; LC/MS: liquid chromatography mass spectrometry B Western blotting detection of PrPSC in the brain. The prion protein (PrP) was detected with mouse monoclonal anti-PrP antibody SAF84 (1:200; Cayman, USA). Lane 1: Proteinase K-untreated whole brain from C57BL/6 mice inoculated with PBS (CTL) at 3 months post-injection. Lane 2: Proteinase K-treated whole brain from C57BL/6 mice inoculated with PBS at 3 months post-injection. Lane 3: Proteinase K-untreated whole brain from C57BL/6 mice inoculated with ME7 scrapie (ME7) at 3 months post-injection. Lane 4 Proteinase K-treated whole brain from C57BL/6 mice inoculated with ME7 scrapie at 3 months post-injection. Lane 5: Proteinase K-untreated whole brain from C57BL/6 mice inoculated with PBS at 7 months post-injection. Lane 6: Proteinase K-treated whole brain from C57BL/6 mice inoculated with PBS at 7 months post-injection. Lane 7: Proteinase K-untreated whole brain from C57BL/6 mice inoculated with ME7 scrapie at 7 months post-injection. Lane 8: Proteinase K-treated whole brain from C57BL/6 mice inoculated with ME7 scrapie at 7 months post-injection. Bars on the left indicate molecular size markers (KDa). -: proteinase K-untreated lanes; +: proteinase K-treated lanes. 


\section{Results}

\section{Detection of PrPsc}

Detailed information on the study workflow is described in Figure 1A. We inoculated the ME7 mouse scrapie strain by intraperitoneal route to mice because of a high success rate of infection, low mortality rate and small inter-individual differences compared to intracranial injection. To detect $\mathrm{PrP}^{\mathrm{Sc}}$ in the brains of non-infected mice and prion-infected mice, we carried out a western blot analysis coupled with a proteinase $\mathrm{K}$ digestion assay (Figure 1B). At 3 months post-injection (early stage), $\mathrm{PrP}^{\mathrm{Sc}}$ was not detected in both non-infected mice and prion-infected mice (Figure 1B, lanes 2, 4). At 7 months post-injection (disease end stage), as expected, the $\operatorname{PrP}^{\mathrm{Sc}}$ band was not detected in the non-infected mice but was detected in the prion-infected mice (Figure 1B, lanes 6 and 8).

\section{Principal component analysis}

To compare the lipidomic data response to prion infection at early and late stage, a principal component analysis was performed using total $\log _{2} \mathrm{FC}$ datasets without threshold restrictions. Although higher dispersion was observed in non-infected mice than in prion-infected mice, each principal component analysis showed wellaligned clusters of the lipidomic data at 3 and 7 months post-infection in prion-infected mice (Figure 2).

\section{Potential lipid biomarkers of prion disease}

Potential lipid biomarkers in the brains of prion-infected mice at 3 and 7 months post-infection were further extracted based on multivariate statistical analyses, with the parameters of the altered lipids satisfying the criteria $\left|\log _{2} \mathrm{FC}\right|>1$ and $P<0.05$. In brief, a total of 43 and 75 compounds showed alterations at 3 and 7 months after ME7 inoculation, respectively (Figure 3A). In the mouse brain at 7 months post-injection, 63 and 12 compounds

\section{ESI+}

(A)

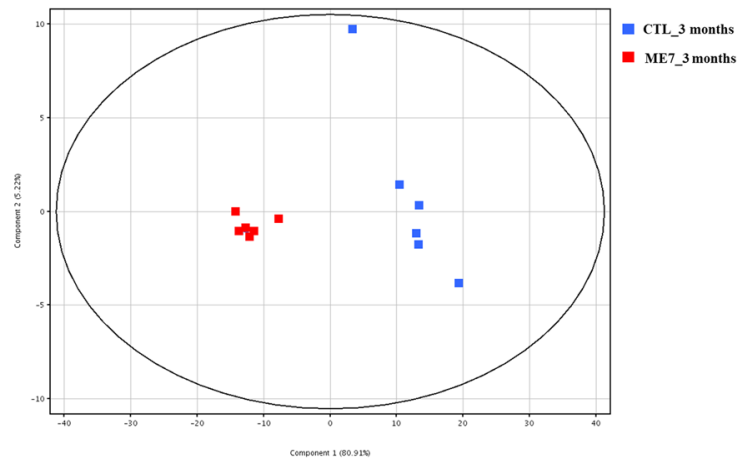

(C)

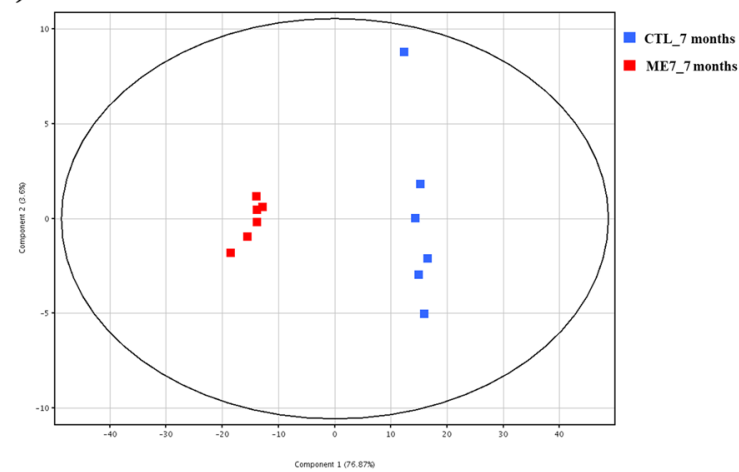

\section{ESI-}

(B)

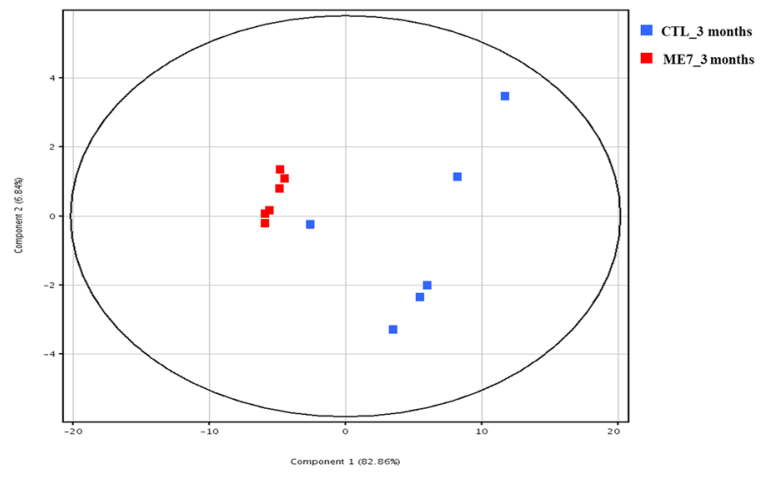

(D)

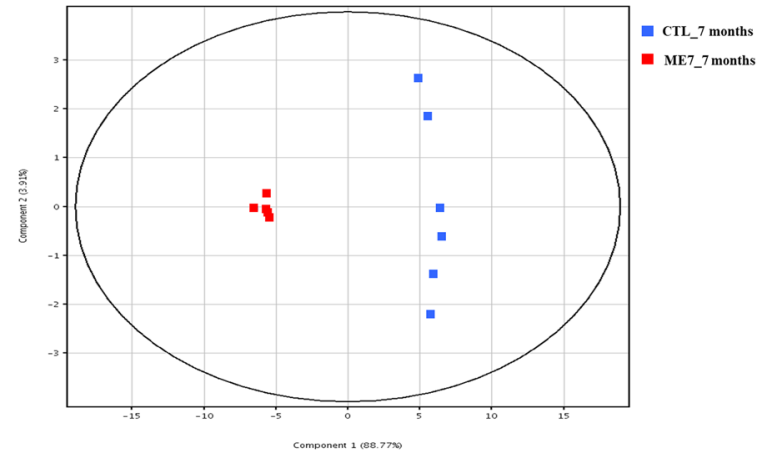

Figure 2 Principal component analysis (PCA) plots of the statistical model for lipidomic data from brain samples in the negative and positive ion modes of HPLC-QTOF/MS. A PCA plot for brain samples at 3 months post-injection in positive ion mode (ESI + ). B PCA plot for brain samples at 3 months post-injection in negative ion mode (ESI-). C PCA plot for brain samples at 7 months post-injection in the positive ion mode. D PCA plot for brain samples at 7 months post-injection in the negative ion mode. Blue dots indicate lipidomic data for the brains of C57BL/6 mice inoculated with PBS (CTL). Red dots indicate lipidomic data for the brains of C57BL/6 mice inoculated with ME7 scrapie (ME7). 


\section{(A)}

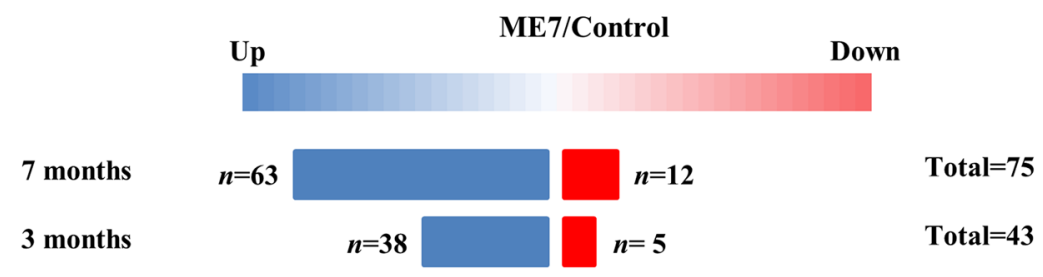

(B)

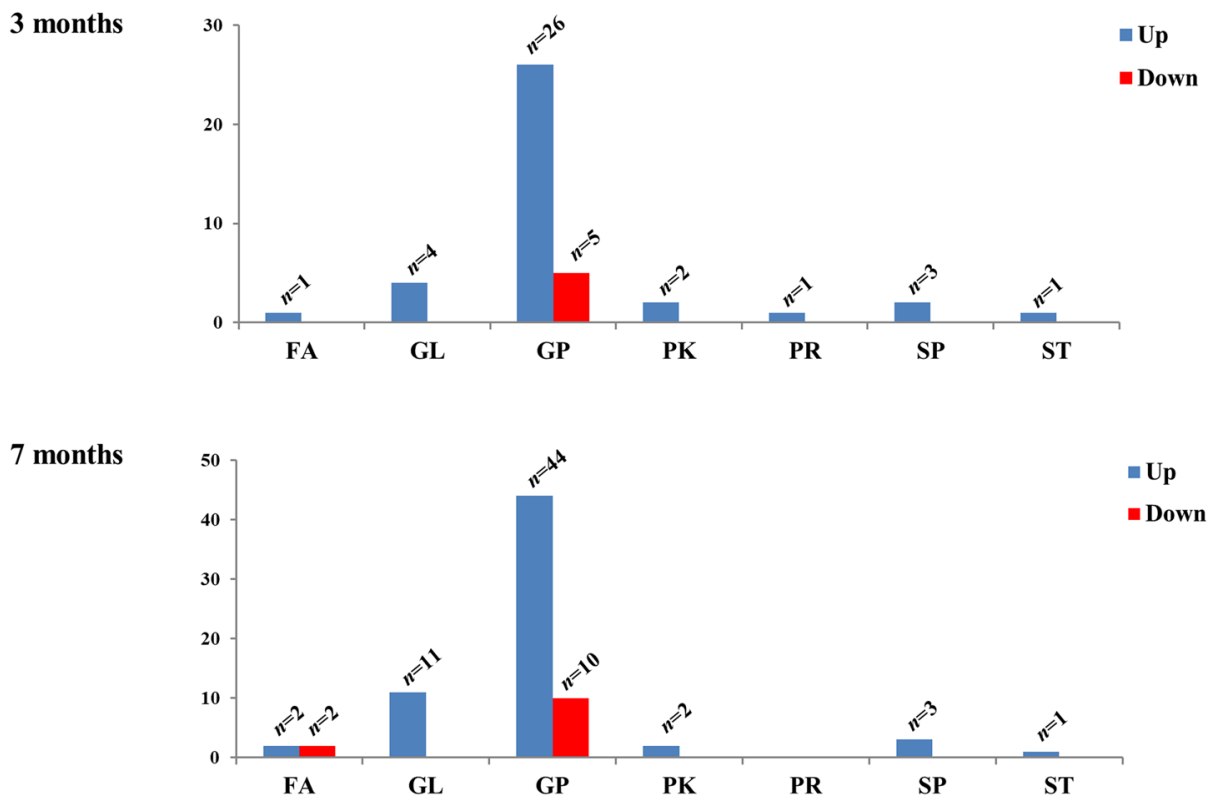

Figure 3 Summary of the lipidomic profiling in prion-infected mice. A Number of lipids showing the level of relative increase and decrease $\left(\left|\log _{2} \mathrm{FC}\right|>1, P<0.05\right)$ in prion-infected mice at 3 and 7 months post-infection. $\mathbf{B}$ Number of lipids showing the level of relative increase and decrease ( $\left(\log _{2} \mathrm{FC}>>1, P<0.05\right)$ according to the lipid class. FA: fatty acids; GL: glycerolipids; GP: glycerophospholipids; PK: polyketides; PR: prenol lipids; SP: sphingolipids; ST: sterol lipids.

were upregulated and downregulated, respectively. In the mouse brain at 3 months post-injection, 38 and 5 compounds were upregulated and downregulated, respectively. We classified altered lipids according to their lipid classes (Figure 3B). A total of 7 lipid classes, i.e., fatty acids, glycerophospholipids, polyketides, prenol lipids, sphingolipids, and sterol lipids, were identified at 3 months post-infection, whereas 6 lipid classes (excluding prenol lipids) were identified in the mouse brain at 7 months post-injection. Notably, approximately $75 \%$ of the total lipid alterations in our analysis consisted of glycerophospholipids at 3 and 7 months post-infection. The alteration of lipids at 3 and 7 months post-infection was visualized by heatmaps (Figures 4,5 ). Detailed information on the alteration of lipids is described in Additional files 1, 2. Notably, 4 lipids including PG [20:0/14:1 (9Z)], PE (19:0/0:0), PE [18:2 (9Z, 12Z)/18:3 (9Z, 12Z, 15Z)] and TG $[20: 3(8 Z, 11 Z, 14 Z) / 20: 5(5 Z, 8 Z, 11 Z, 14 Z$, 17Z)/22:6 (4Z, 7Z, 10Z, 13Z, 16Z, 19Z)] [iso6] were commonly upregulated in the brain of prion-infected mice at both 3 and 7 months post-infection.

\section{Pathway analysis}

To investigate the lipid alteration-related pathways, we performed a pathway analysis by LIPEA based on the KEGG database source (Figure 6). The heatmap showed the top 20 signaling pathways enriched at 7 months post-infection and their enrichment at 3 months postinfection. As expected based on the alterations in lipids, sphingolipid metabolism and signaling pathways 

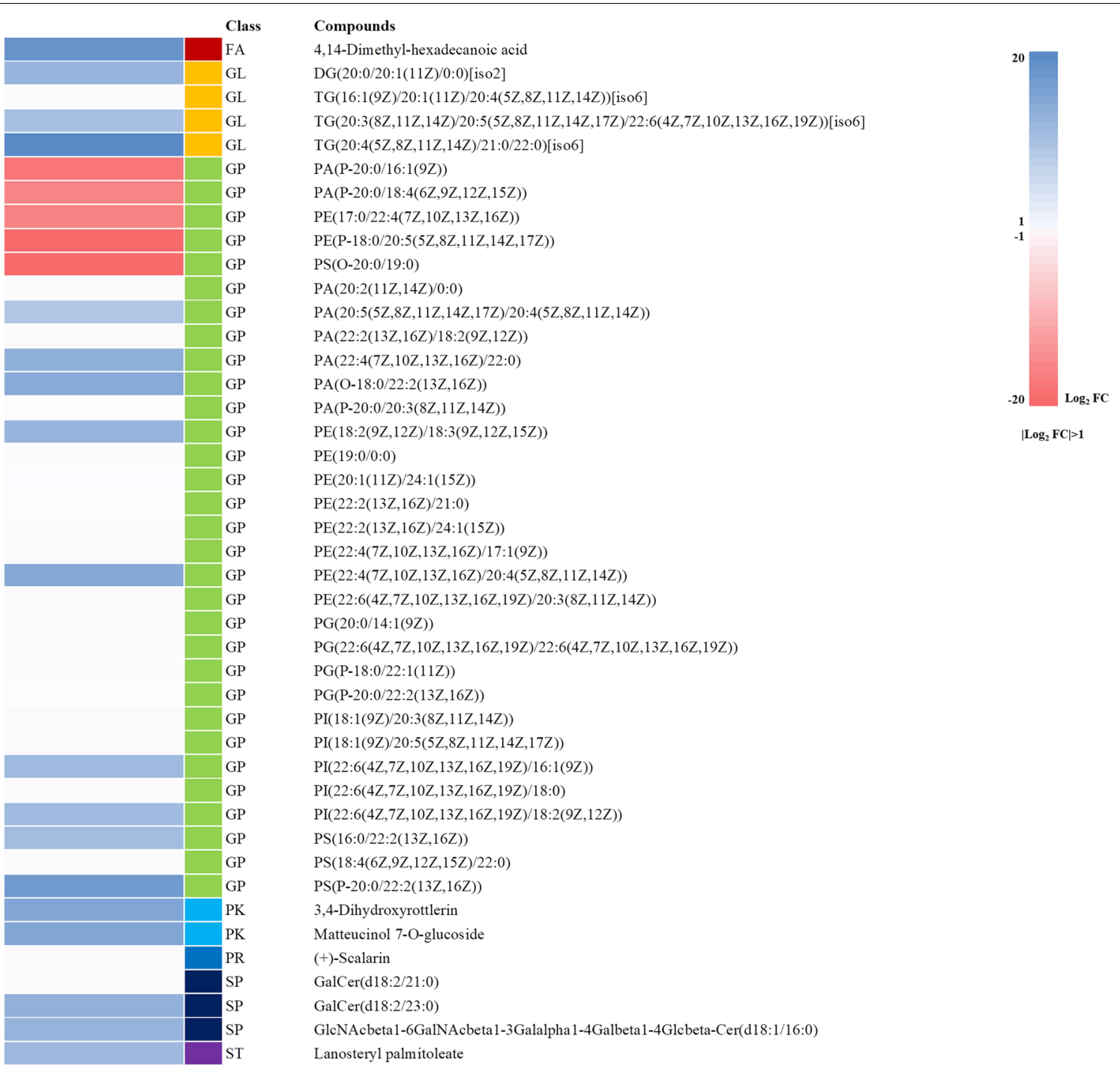

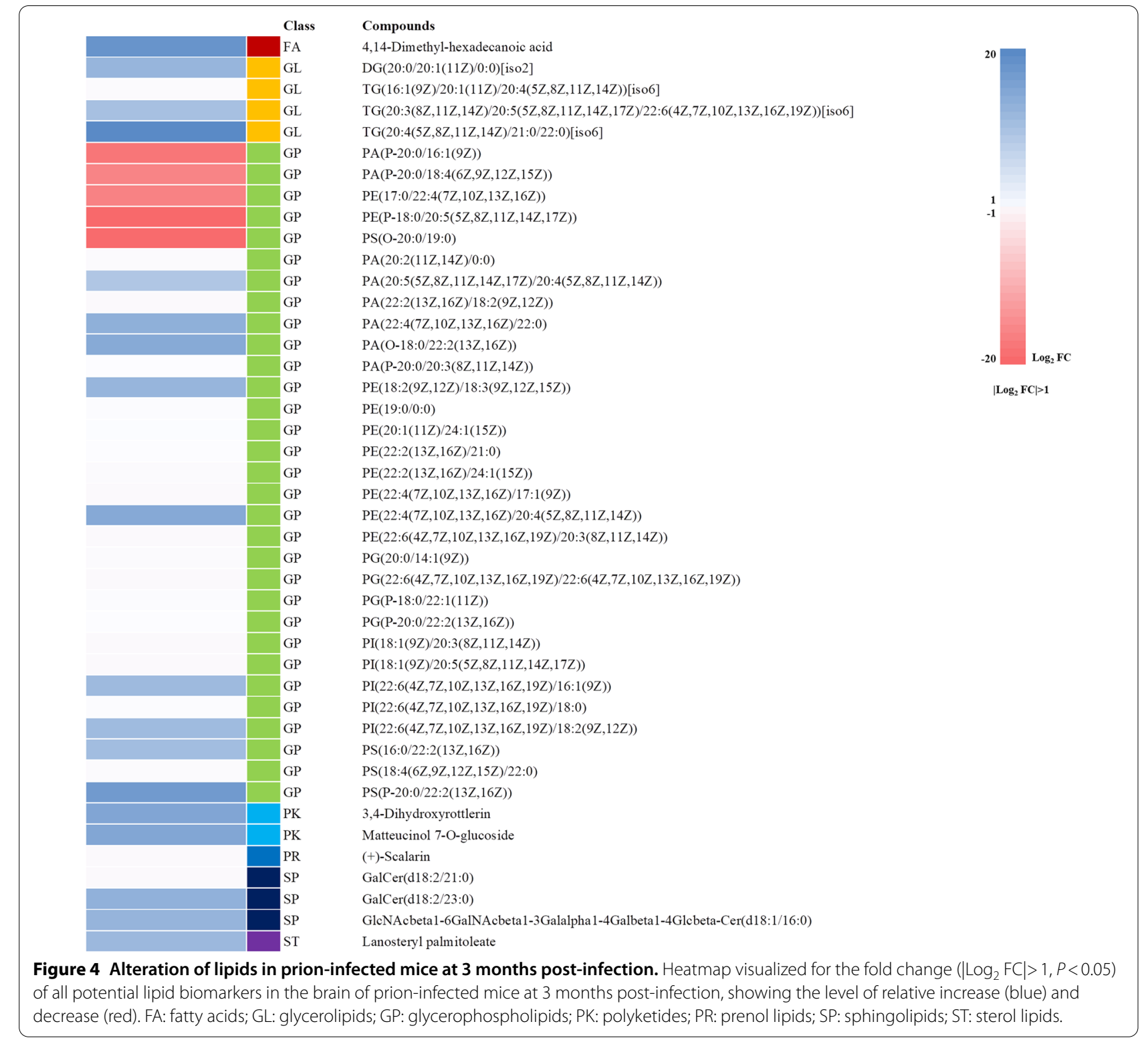

and glycerophospholipid and choline metabolismrelated pathways were commonly enhanced at both 3 and 7 months post-infection. Interestingly, the brains at 3 and 7 months post-infection from prion-infected mice showed enhancement of GPI-anchor biosynthesis, autophagy and necroptosis-related pathways.

\section{Discussion}

In the present study, although we did not detect $\operatorname{Pr} \mathrm{P}^{\mathrm{Sc}}$ in ME7 scrapie-injected mice at 3 months (Figure 1B), global lipidomic changes were found in the early stage of prion disease (Figure 4). However, since the detection limit of $\mathrm{PrP}^{\mathrm{Sc}}$ by western blot analysis is relatively low with approximately $4 \mathrm{ng}, \mathrm{PrP}^{\mathrm{Sc}}$ may not be detected in prioninfected mice at 3 months post-infection. Thus, to resolve these limitations, further investigations using a more sensitive assays including protein misfolding cyclic amplification (PMCA) and real-time quaking-induced conversion (RT-QuIC) are needed. In addition, we also identified a total of 43 and 75 novel biomarkers at 3 and 7 months, respectively (Figures 3A, 4 and 5). In a previous study, the single lipid substance phosphatidylethanolamine accelerated the conversion of $\operatorname{PrP}^{\mathrm{C}}$ to $\operatorname{PrP}^{\mathrm{Sc}}$; thus, the altered lipids found in this study need to be investigated to further understand whether they participate to the disease and/or the conversion processes [25]. Notably, 4 lipids including 

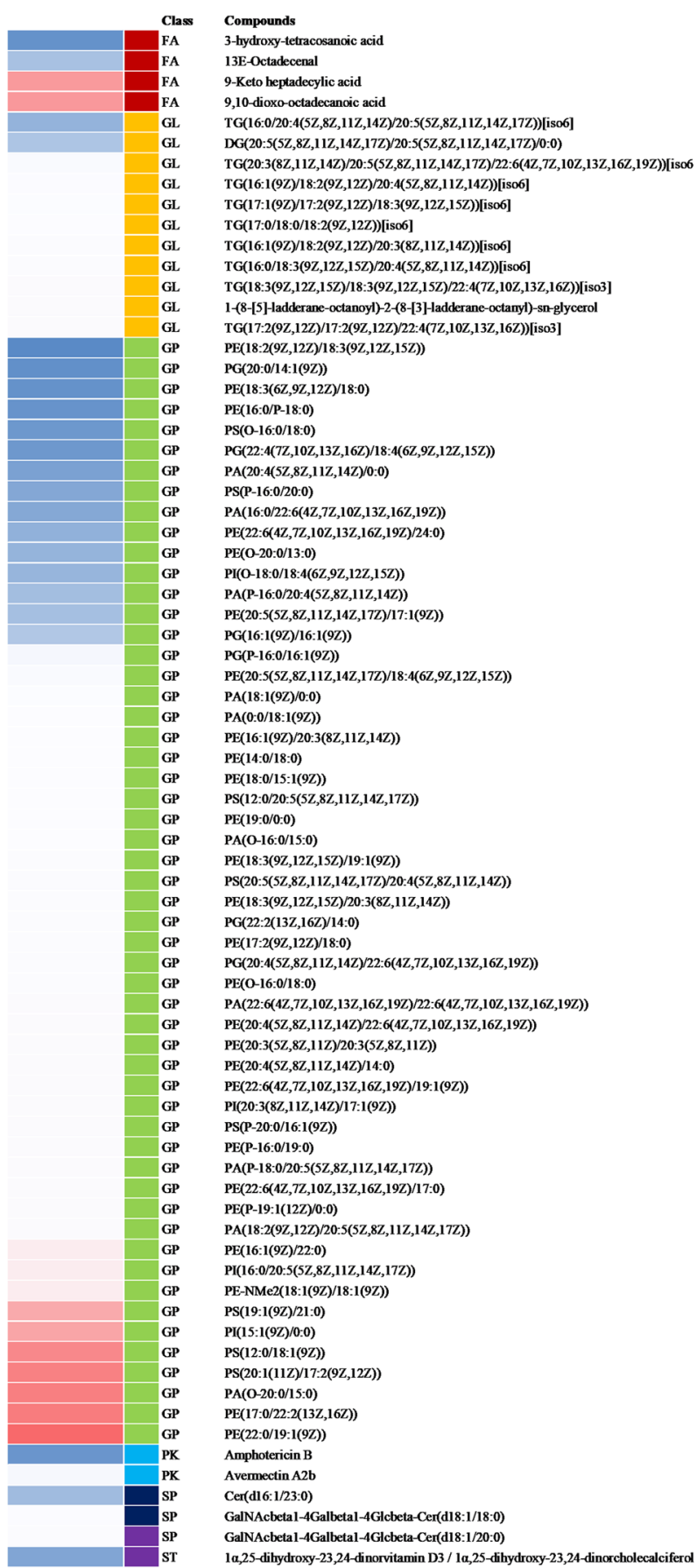

1a,25-dihydroxy-23,24-dinorvitamin D3 / 1a,25-dihydroxy-23,24-dinorcholecaleiferol

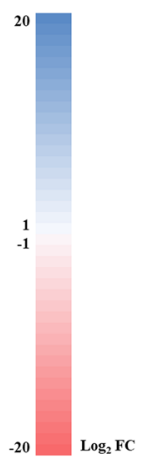

$\left|\log _{2} \mathbf{F C}\right|>1$

Figure 5 Alteration of lipids in prion-infected mice at 7 months post-infection. Heatmap visualized for the fold change $\left(\left|\log _{2} F C\right|>1, P<0.05\right)$ of all potential lipid biomarkers in the brain of prion-infected mice at 7 months post-infection, showing the level of relative increase (blue) and decrease (red). FA: fatty acids; GL: glycerolipids; GP: glycerophospholipids; PK: polyketides; PR: prenol lipids; SP: sphingolipids; ST: sterol lipids.

PG [20:0/14:1(9Z)], PE (19:0/0:0), PE [18:2 (9Z, 12Z)/18:3 (9Z, 12Z, 15Z)] and TG [20:3 (8Z, 11Z, 14Z)/20:5 (5Z, $8 Z, 11 Z, 14 Z, 17 Z) / 22: 6(4 Z, 7 Z, 10 Z, 13 Z, 16 Z, 19 Z)]$ [iso6] were commonly upregulated in prion-infected mice at both 3 and 7 months post-infection. These lipids are worth investigating the possibility as potential biomarkers for prion disease in the future. In addition, we also found several lipid-related pathways in our animal model of prion disease (Figure 6). Among them, we found that sphingolipid metabolism and signaling pathways and the 
Sphingolipid metabolism

Glycerophospholipid metabolism

Sphingolipid signaling pathway

Choline metabolism in cancer

Glycosylphosphatidylinositol (GPI)-anchor biosynthesis

Autophagy

Necroptosis

Leishmaniasis

Fat digestion and absorption

Systemic lupus erythematosus

Basal cell carcinoma

Retrograde endocannabinoid signaling

AGE-RAGE signaling pathway in diabetic complications

Aldosterone synthesis and secretion

Cholesterol metabolism

Pancreatic cancer

Glycine, serine and threonine metabolism

Butanoate metabolism

Neurotrophin signaling pathway

GnRH signaling pathway
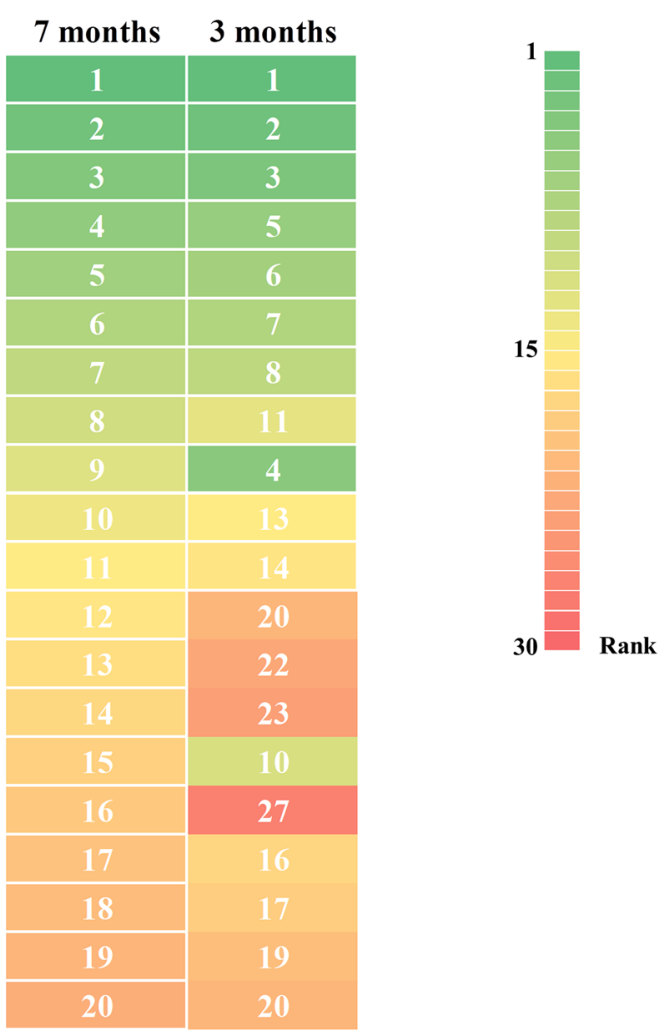

Figure 6 Heatmap displaying the top 20 signaling pathways enriched at 7 months post-infection and their enrichment at 3 months post-infection. Numbers and colors indicate the ranking (high: green; middle: yellow; low: red) of the respective signaling pathways.

GPI-anchor biosynthesis pathway are related to lipidomic profiling. Since sphingolipids and GPI anchors are major lipid rafts components, which are prerequisites for the conversion of $\operatorname{PrP}^{\mathrm{Sc}}$, this result was noteworthy $[19,20]$. In a previous study, sphingolipid depletion increased the conversion of $\mathrm{PrP}^{\mathrm{C}}$ into $\mathrm{PrP}^{\mathrm{Sc}}$ in neuroblastoma cells [25]. In addition, several inhibitors of cellular cholesterol, the major lipid rafts component, were associated with a decrease in $\mathrm{PrP}^{\mathrm{Sc}}$ formation [16-18]. Thus, the investigation of the relationship between altered levels of sphingolipids, GPIanchor-related lipids and prion diseases is highly desirable in the future.

Although the cholesterol metabolism pathway showed a relatively low rank in prion-infected mice, the acceleration effect of cholesterol in $\mathrm{PrP}^{\mathrm{Sc}}$ formation was evident in previous studies [26, 27]. In addition, cholesterol metabolism was changed in prion-infected neuronal cells and GT-1 cells and cholesterol biosynthesis was upregulated in primary hippocampal neurons. Furthermore, cholesterol accumulated in 22L scrapie-infected N2a cells and the mouse brain [26, 27]. We also found that 2 lipids in sterol class including lanosteryl palmitoleate and 1 $\alpha, 25$-dihydroxy-23,24-dinorvitamin D3/1 $\alpha, 25$ dihydroxy-23,24-dinorcholecalciferol were upregulated in ME7 scrapie -infected mice at 3 and 7 months postinfection, respectively. These results indicate that our findings correlated with previous studies despite the use of different prion strains. Thus, further investigation of the association between prion diseases and cholesterol metabolism pathway-related altered lipids identified in this study is warranted.

The exact mechanism of lipid alteration in cell/organelle membranes in prion diseases remains elusive, however, previous studies suggested that the conversion of $\mathrm{PrP}^{\mathrm{C}}$ into $\mathrm{PrP}^{\mathrm{Sc}}$ occurs on lipid rafts and $\mathrm{PrP}^{\mathrm{Sc}}$-induced signal affects cell viability via the lipid-mediated cellular signal pathway $[28,29]$. In addition, neuronal loss, astrocytosis and activated-microglia are predefined prion symptoms and also are related to global alteration of lipids [30, 31]. Thus, cell type and cellular compartmentspecific lipidomic analyses are needed in the future to improve our knowledge of prion pathogenic mechanisms.

In the present study, we also found that the autophagy pathway is related to lipidomic profiling (Figure 6). In detail, 4,14-Dimethyl-hexadecanoic and 9,10-dioxo-octadecanoic acid were interpreted to be associated with the autophagy-related pathway (data not shown). Prion diseases are caused by the accumulation of misfolded PrP, 
and the autophagy signaling pathway may be initially activated to eliminate $\operatorname{PrP}^{\mathrm{Sc}}$. Conversely, the activation of autophagy beyond cellular capacity induces dysfunctional autophagy and may contribute to the pathophysiology of the disease [32, 33]. Our findings indicate that both the early and late stages of the disease pathogenesis are commonly related to autophagy-related pathways at the lipid level. In addition, the necroptosis pathway has also been shown to be related to lipidomic profiling. In a previous study, a cell culture model of prion disease showed elevated biomarkers of necroptosis. Our findings provide supporting data that prion diseases are also involved in necroptosis at the lipid level.

In a recent study, a lipidomic analysis of a mouse model of Alzheimer's disease showed that it is strongly associated with sphingolipid metabolism, glycerophospholipid metabolism and arachidonic acid metabolism pathways [34]. Interestingly, our mouse model of prion disease displayed increased sphingolipid metabolism and glycerophospholipid metabolism, which is consistent with that of Alzheimer's disease; however, the arachidonic acid metabolism pathways did not show any increase in the brain of prion-infected mice at 3 months postinfection, and these pathways showed a very low rank in prion-infected mice at 7 months post-infection (data not shown). Conversely, our mouse model of prion disease presented unique pathways compared to those of Alzheimer's disease, including choline metabolism and GPI-anchor biosynthesis (Figure 6). Prion diseases and Alzheimer's disease are neurodegenerative diseases caused by misfolded proteins, $\mathrm{PrP}^{\mathrm{Sc}}$ and amyloid beta, and they show similar pathophysiological features, including transmissibility, endoplasmic reticulum stress and autophagy-related responses [32, 35, 36]. In addition, $13 \%$ of clinically diagnosed Alzheimer's disease patients were rediagnosed as having human prion disease postmortem [37]. These studies indicate that there is difficulty in distinguishing between prion diseases and Alzheimer's disease and that there is a need for a clear understanding of the differences between these two diseases. Thus, the unique lipidomic mechanism identified in the present study may be characteristic to prion disease. In addition, 4 lipids including PG [20:0/14:1 (9Z)], PE (19:0/0:0), PE [18:2 (9Z, 12Z)/18:3 (9Z, 12Z, 15Z)] and TG [20:3 (8Z, $11 \mathrm{Z}, 14 \mathrm{Z}) / 20: 5(5 \mathrm{Z}, 8 \mathrm{Z}, 11 \mathrm{Z}, 14 \mathrm{Z}, 17 \mathrm{Z}) / 22: 6$ (4Z, 7Z, $10 Z, 13 Z, 16 Z, 19 Z)$ ] [iso6] were commonly upregulated in prion-infected mice at both 3 and 7 months post-infection. Since these lipids were not altered in Alzheimer's disease and specifically altered in the prion-infected animal model, these lipids may be considered as potential specific biomarkers of prion diseases. However, since the present study was performed using only one prion strain (ME7), these results could be prion strain-specific.
Further validation with other prion strains including 22L, RML and 139A is highly desirable in the future.

In the present study, we first carried out a large-scale lipidomic profiling in an animal model of prion disease and identified a total of 43 and 75 novel potential biomarkers at 3 and 7 months post-infection, respectively. Notably, approximately $75 \%$ of the total lipid alterations at 3 and 7 months post-infection were accounted for by glycerophospholipids. In addition, altered lipids between non-infected and prion-infected mice are related to lipid raft-related pathways. In the present study, we found novel potential biomarkers and therapeutic targets of prion disease. To the best of our knowledge, this was the first large-scale lipidomic profiling of prion disease.

\section{Abbreviations}

PrP: Prion protein; PrPsc: Abnormal prion protein; PrPC: Normal prion protein; LC/MS: Liquid chromatography mass spectrometry; GPI:

Glycosylphosphatidylinositol.

\section{Supplementary Information}

The online version contains supplementary material available at https://doi. org/10.1186/s13567-021-00975-1.

Additional file 1. Comparison of lipid between non-infected and prion-infected mice at 3 months post-infection.

Additional file 2. Comparison of lipid between non-infected and prion-infected mice at 7 months post-infection.

\section{Acknowledgements}

Yong-Chan Kim was supported by the BK21 Plus Program in the Department of Bioactive Material Sciences.

\section{Authors' contributions}

Y-CK and B-HJ conceived and designed the experiment. Y-CK and JL performed the experiments. Y-CK, JL and B-HJ analyzed the data. Y-CK, D-WL and $\mathrm{B}-\mathrm{HJ}$ wrote the paper. All authors read and approved the final manuscript.

\section{Funding}

This research was supported by the Basic Science Program through the National Research Foundation (NRF) of Korea funded by the Ministry of Education, Science and Technology (2018R1D1A1B07048711). This research was supported by the Basic Science Research Program through the National Research Foundation (NRF) of Korea funded by the Ministry of Education (2017R1A6A1A03015876). This work was supported by NRF (National Research Foundation of Korea) Grant funded by the Korean Government (NRF-

2019-Fostering Core Leaders of the Future Basic Science Program/Global Ph.D. Fellowship Program).

\section{Declarations}

\section{Ethics approval and consent to participate}

All efforts were made to minimize the number of animals used and their suffering. All experimental procedures were accredited by the Institute of Animal Care and Use Committee of Jeonbuk National University (JBNU 2020-080).

\section{Competing interests}

The authors declare that they have no competing interests. 


\section{Author details}

${ }^{1}$ Korea Zoonosis Research Institute, Jeonbuk National University, Iksan, Jeonbuk 54531, Republic of Korea. ${ }^{2}$ Department of Bioactive Material Sciences and Institute for Molecular Biology and Genetics, Jeonbuk National University, Jeonju, Jeonbuk 54896, Republic of Korea. ${ }^{3}$ Metabolomics Research Center for Functional Materials, Kyungsung University, Busan 48434, Republic of Korea. ${ }^{4}$ Department of Bio-Safety, Kyungsung University, Busan 48434 Republic of Korea.

Received: 20 April 2021 Accepted: 30 June 2021

Published online: 21 July 2021

\section{References}

1. Prusiner SB (1998) Prions. Proc Natl Acad Sci U S A 95:13363-13383

2. Prusiner SB (1998) The prion diseases. Brain Pathol 8:499-513

3. Kim YC, Won SY, Jeong MJ, Jeong BH (2021) Absence of proteinase K-resistant PrP in Korean Holstein cattle carrying potential bovine spongiform encephalopathy-related E211K somatic mutation. Transbound Emerg Dis In press.

4. Won SY, Kim YC, Jeong BH (2020) First report of the potential bovine spongiform encephalopathy (BSE)-related somatic mutation E211K of the prion protein gene (PRNP) in cattle. Int J Mol Sci 21:4246

5. Jeong BH, Kim YS (2014) Genetic studies in human prion diseases. J Korean Med Sci 29:623-632

6. Kim YC, Won SY, Jeong BH (2020) Identification of prion disease-related somatic mutations in the prion protein gene (PRNP) in cancer patients. Cells 9:1480

7. Castle AR, Gill AC (2017) Physiological functions of the cellular prion protein. Front Mol Biosci 4:19

8. Prusiner SB (1991) Prion biology and diseases. Harvey Lect 87:85-114

9. Wulf MA, Senatore A, Aguzzi A (2017) The biological function of the cellular prion protein: an update. BMC Biol 15:34

10. Sigurdson CJ, Bartz JC, Glatzel M (2019) Cellular and molecular mechanisms of prion disease. Annu Rev Pathol 14:497-516

11. Kovacs GG, Budka H (2009) Molecular pathology of human prion diseases. Int J Mol Sci 10:976-999

12. Gambetti P, Cali I, Notari S, Kong Q, Zou WQ, Surewicz Q (2011) Molecular biology and pathology of prion strains in sporadic human prion diseases. Acta Neuropathol 121:79-90

13. Elfrink K, Nagel-Steger $L$, Riesner D (2007) Interaction of the cellular prion protein with raft-like lipid membranes. Biol Chem 388:79-89

14. Campana V, Sarnataro D, Zurzolo C (2005) The highways and byways of prion protein trafficking. Trends Cell Biol 15:102-111

15. Cui HL, Guo B, Scicluna B, Coleman BM, Lawson VA, Ellett L, Meikle PJ, Bukrinsky M, Mukhamedova N, Sviridov D, Hill AF (2014) Prion infection impairs cholesterol metabolism in neuronal cells. J Biol Chem 289:789-802

16. Taraboulos A, Scott M, Semenov A, Avrahami D, Laszlo L, Prusiner SB (1995) Cholesterol depletion and modification of $\mathrm{COOH}$-terminal targeting sequence of the prion protein inhibit formation of the scrapie isoform. J Cell Biol 129:121-132

17. Marella M, Lehmann S, Grassi J, Chabry J (2002) Filipin prevents pathological prion protein accumulation by reducing endocytosis and inducing cellular PrP release. J Biol Chem 277:25457-25464

18. Bate C, Salmona M, Diomede L, Williams A (2004) Squalestatin cures prion-infected neurons and protects against prion neurotoxicity. J Biol Chem 279:14983-14990
19. McNally KL, Ward AE, Priola SA (2009) Cells expressing anchorless prion protein are resistant to scrapie infection. J Virol 83:4469-4475

20. Puig B, Puig B, Altmeppen HC, Linsenmeier L, Chakroun K, Wegwitz F, Piontek UK, Tatzelt J, Bate C, Magnus T, Glatzel M (2019) GPI-anchor signal sequence influences $\operatorname{PrP}^{C}$ sorting, shedding and signalling, and impacts on different pathomechanistic aspects of prion disease in mice. PLoS Pathog 15:1007520

21. Deleault NR, Piro JR, Walsh DJ, Wang F, Ma J, Geoghegan JC, Supattapone S (2012) Isolation of phosphatidylethanolamine as a solitary cofactor for prion formation in the absence of nucleic acids. Proc Natl Acad Sci U S A 109:8546-8551

22. Feraudet $C$, Morel N, Simon S, Volland H, Frobert $Y$, Creminon C, Vilette D, Lehmann S, Grassi J (2005) Screening of 145 anti-PrP monoclonal antibodies for their capacity to inhibit PrPSc replication in infected cells. J Biol Chem 280:11247-11258

23. Bligh EG, Dyer WJ (1959) A rapid method of total lipid extraction and purification. Can J Biochem Physiol 37:911-917

24. LIPEA. https://lipea.biotec.tu-dresden.de/home. Assessed 18 Nov 2020

25. Naslavsky N, Shmeeda H, Friedlander G, Yanai A, Futerman AH, Barenholz Y, Taraboulos A (1999) Sphingolipid depletion increases formation of the scrapie prion protein in neuroblastoma cells infected with prions. J Biol Chem 274:20763-20771

26. Bach C, Gilch S, Rost R, Greenwood AD, Horsch M, Hajj GN, Brodesser S, Facius A, Schadler S, Sandhoff K, Beckers J, Leib-Mösch C, Schätzl HM, Vorberg I (2009) Prion-induced activation of cholesterogenic gene expression by Srebp2 in neuronal cells. J Biol Chem 284:31260-31269

27. Mok SW, Thelen KM, Riemer C, Bamme T, Gultner S, Lutjohann D, Baier $M$ (2006) Simvastatin prolongs survival times in prion infections of the central nervous system. Biochem Biophys Res Commun 348:697-702

28. Lewis V, Hooper NM (2011) The role of lipid rafts in prion protein biology. Front Biosci 16:151-168

29. Taylor DR, Hooper NM (2006) The prion protein and lipid rafts. Mol Membr Biol 23:89-99

30. Loving BA, Bruce KD (2020) Lipid and lipoprotein metabolism in microglia. Front Physiol 11:393

31. Barber CN, Raben DM (2019) Lipid metabolism crosstalk in the brain: glia and neurons. Front Cell Neurosci 13:212

32. Hao Z, Wang G (2020) Autophagy and prion disease. Adv Exp Med Biol 1207:75-85

33. Lopez-Perez O, Badiola JJ, Bolea R, Ferrer I, Llorens F, Martin-Burriel I (2020) An update on autophagy in prion diseases. Front Bioeng Biotechnol 8:975

34. Chan RB, Oliveira TG, Cortes EP, Honig LS, Duff KE, Small SA, Wenk MR, Shui G, Di Paolo G (2012) Comparative lipidomic analysis of mouse and human brain with Alzheimer disease. J Biol Chem 287:2678-2688

35. Scheckel C, Aguzzi A (2018) Prions, prionoids and protein misfolding disorders. Nat Rev Genet 19:405-418

36. Chen S, Zhou Q, Ni Y, Le W (2020) Autophagy and Alzheimer's disease. Adv Exp Med Biol 1207:3-19

37. Manuelidis EE, Manuelidis L (1989) Suggested links between different types of dementias: Creutzfeldt-Jakob disease, Alzheimer disease, and retroviral CNS infections. Alzheimer Dis Assoc Disord 3:100-109

\section{Publisher's Note}

Springer Nature remains neutral with regard to jurisdictional claims in published maps and institutional affiliations. 